\title{
Autoevaluación de prácticas inclusivas docentes en atención a la población con aptitudes sobresalientes
}

Self-evaluation of inclusive practices in teaching the gifted population

\author{
Volumen 21, Número 2 \\ Mayo - Agosto \\ pp. $1-24$
}

Norma Guadalupe Márquez Cabellos

Adriana Isabel Andrade Sánchez

Ismael García Cedillo

\section{Citar este documento según modelo APA}

Márquez Cabellos, Norma Guadalupe., Andrade Sánchez, Adriana Isabel., y García Cedillo, Ismael. (2021). Autoevaluación de prácticas inclusivas docentes en atención a la población con aptitudes sobresalientes. Revista Actualidades Investigativas en Educación, 21(2), 1-24. Doi. 10.15517/aie.v21i2.46759 


\title{
Autoevaluación de prácticas inclusivas docentes en atención a la población con aptitudes sobresalientes
}

Self-evaluation of inclusive practices in teaching the gifted population

\author{
Norma Guadalupe Márquez Cabellos ${ }^{1}$ \\ Adriana Isabel Andrade Sánchez ${ }^{2}$ \\ Ismael García Cedillo ${ }^{3}$
}

Resumen: Las prácticas inclusivas en el contexto escolar implican el uso de una variedad de estrategias por parte del profesorado para asegurar el aprendizaje y la participación de todo el alumnado. Esta investigación tuvo como objetivo analizar la autoevaluación de prácticas inclusivas del profesorado que atiende a adolescentes con aptitudes sobresalientes de una escuela secundaria con modalidad de semi-internado del estado de Colima, México. Se diseñó un estudio cuantitativo, transversal, con alcance descriptivo, donde participaron 34 docentes que laboraron en el ciclo escolar 2019-2020. Los datos se recolectaron mediante la Guía de Evaluación de Prácticas Inclusivas en el Aula Regular -GEPIA- Formato de Autorreporte, la Escala de Autoeficacia para Implementar Prácticas Inclusivas y el Cuestionario de Estrategias para Fortalecer el Aprendizaje. Para la fiabilidad de los instrumentos se calculó el Alpha de Cronbach y para el tratamiento de los datos se aplicaron técnicas de la estadística descriptiva e inferencial, así como la $U$ de Mann-Whitney. Los resultados indican que las personas docentes se autoevalúan con buenas prácticas inclusivas, por la implementación de estrategias diversificadas en la metodología y la evaluación, así como por la promoción del trabajo colaborativo con la comunidad escolar y la vinculación con padres de familia. Sin embargo, el profesorado menciona necesitar capacitación, actualización y formación en temas relacionados con la educación inclusiva y la atención a las aptitudes sobresalientes. Asimismo, indican que requieren mayores recursos para promover el aprendizaje entre sus estudiantes. Se concluye que las prácticas inclusivas valoran positivamente la diversidad escolar, conducen a la movilización de acciones pedagógicas para el logro de la calidad en el aprendizaje y la satisfacción de necesidades educativas de la población sobresaliente.

Palabras clave: prácticas inclusivas, educación inclusiva, educación secundaria, aptitudes sobresalientes.

Abstract: Inclusive practices in the school context involve the use of a variety of strategies by teachers to ensure the learning and participation of all students. The objective of this research was to analyze the self-evaluation of inclusive practices of teachers who serve adolescents with gifted abilities from a secondary school with a semiboarding modality in the state of Colima, Mexico. A quantitative, cross-sectional study was designed, with a descriptive scope, where 34 teachers who worked in the 2019-2020 school year participated. Data were collected through the Guide for the Evaluation of Inclusive Practices in the Regular Classroom -GEPIA-Self-Report Format, the Self-Efficacy Scale to Implement Inclusive Practices and the Questionnaire on Strategies to Strengthen Learning. For the reliability of the instruments, Cronbach's Alpha was calculated and descriptive and inferential statistics techniques were applied for data treatment, as well as the Mann-Whitney $U$. The results indicate that teachers selfevaluate with inclusive good practices, by implementing diversified strategies in methodology and evaluation, as well as by promoting collaborative work with the school community and linking with parents. However, teachers mention that they need training, updating and training on issues related to inclusive education and attention to gifted skills. They also indicate that they require more resources to promote learning among their students. It is concluded that inclusive practices positively value school diversity, lead to the mobilization of pedagogical actions for the achievement of quality in learning and the satisfaction of educational needs of the gifted population.

Key words: inclusive practices, inclusive education, secondary education, outstanding attitudes.

\footnotetext{
1 Universidad de Colima, Colima, México. Dirección electrónica: norma marquez@ucol.mx ORCID: https://orcid.org/0000-0001-5466-2681

2 Universidad de Colima, Colima, México. Dirección electrónica: isa andrade@ucol.mx ORCID: https://orcid.org/0000-0001-9790-5719

${ }^{3}$ Universidad Autónoma de San Luis Potosi, San Luis Potosí, México. Dirección electrónica: ismaelgace@yahoo.com.mx ORCID: https://orcid.org/0000-0002-4820-5633
}

Artículo recibido: 27 de noviembre, 2020

Enviado a corrección: 3 de marzo,2021

Aprobado: 19 de abril, 2021 


\section{Introducción}

En la actualidad, las escuelas $y$, en particular, el colectivo docente, se ven en la necesidad de transformarse para responder a las diversas necesidades educativas del alumnado. De cara a una sociedad heterogénea, los métodos de enseñanza homogeneizadores quedan lejos de la realidad social que se vive. Frente a esta realidad se requiere de prácticas docentes que fomenten una pedagogía inclusiva.

Orozco y Moriña (2019) puntualizan que las prácticas inclusivas deben sustentarse en múltiples y variadas estrategias metodológicas, una evaluación y autoevaluación auténticas, así como un rol docente centrado en el acompañamiento, donde el alumnado sea el protagonista de su propio aprendizaje. En este contexto, el aula se convierte en un espacio donde se concretan aprendizajes significativos que emergen de prácticas docentes inclusivas, comprometidas e innovadoras, gestándose, además, un compromiso y trabajo colaborativo entre la escuela y la comunidad. De esta manera, las prácticas inclusivas tratan de asegurar que las actividades que se llevan a cabo en el aula faciliten y promuevan el aprendizaje y la participación de todo el alumnado, partiendo de sus competencias y potencialidades. Para lograr lo anterior, tal como lo refieren Booth y Ainscow (2015), el profesorado debe movilizar los recursos del centro y de la comunidad para mantener la participación y el aprendizaje activo de todos y todas sus estudiantes.

Atender la diversidad escolar ha sido un reto para el Sistema Educativo Mexicano, puesto que parte de la premisa de que toda persona tiene derecho a recibir una educación orientada a desarrollar al máximo sus capacidades, que le permita construir y orientarse al logro de su proyecto de vida. Hacer efectivo este derecho implica asegurar tres principios fundamentales: integridad, equidad y excelencia educativa. Estos principios implican una educación que garantiza el aprendizaje y la participación de la población estudiantil porque se intersectan las oportunidades y condiciones para atender la diversidad escolar desde la singularidad y el respeto (Secretaría de Educación Pública [SEP], 2019).

Últimamente, los centros educativos de muchos países han puesto la mirada en la población infantil y juvenil que presenta alguna discapacidad o trastorno, así como aquella que presenta aptitudes sobresalientes ${ }^{4}$. México no es la excepción, por ello ha suscrito convenios internacionales y ha propuesto en su Constitución Política de los Estados Unidos Mexicanos

\footnotetext{
${ }^{4}$ El término aptitudes sobresalientes fue adoptado por el sistema educativo mexicano, con el fin de utilizar una terminología común en la comunidad docente al implementar la propuesta de intervención educativa en la atención a la población sobresaliente (SEP, 2006).
} 
(Cámara de Diputados del H. Congreso de la Unión de México, 2019a) y en su Ley General de Educación (Cámara de Diputados del H. Congreso de la Unión, 2019b), una educación inclusiva que responda a la diversidad del estudiantado en las aulas, que potencie y promueva al máximo sus capacidades y habilidades con apoyos y recursos didácticos y profesionales.

Para efectos de la presente investigación, se centra la atención en analizar la autoevaluación de prácticas inclusivas del profesorado que interviene con la población que presenta aptitudes sobresalientes (AS). La acción educativa con esta población, en las aulas regulares, se produce en el marco de una estructura organizativa definida por la Secretaría de Educación Pública (SEP), que, en el año 2006, anunció la estrategia de intervención educativa. Sin embargo, esta estrategia de intervención tiene sus áreas de oportunidad, tal como lo refieren Covarrubias y Marín (2015), al puntualizar que requiere de una profunda revisión de los mecanismos empleados en la operatividad, en la capacitación docente y en la intervención directa con la población estudiantil que presenta aptitudes sobresalientes. En otras palabras, el alumnado con AS no parece estar recibiendo una educación acorde con sus potencialidades en la escuela regular.

Con mucha frecuencia se piensa que la educación especial está orientada a la atención del alumnado con discapacidad o trastornos de algún tipo. Se deja de lado que también la población sobresaliente requiere de una atención educativa en la que se pongan en juego estrategias diferenciadas para su apropiada atención. Por ello, su atención se ha responsabilizado a la educación regular con el apoyo de la educación especial (Ley General de Educación, Artículo 41). No obstante, esta población permanece en una situación de mayor invisibilidad pues, mientras que la discusión actual, promovida por investigadores de la educación inclusiva radical (Booth y Ainscow, 2015, Blanco, 2006) se centra en la necesidad de que toda la población estudiantil asista a la misma escuela, no a escuelas especiales, los educandos que presentan AS (quienes generalmente han sido atendidos en las escuelas regulares) no son tomados en cuenta. Como dicen Juárez, Comboni y Garnique (2010), "en México hay pocas instituciones que atiendan a estas necesidades educativas especiales y pocas escuelas cuentan con maestros preparados para detectar a este tipo de alumnos" (p. 63).

Pese a lo anterior, en la política educativa existe una mayor conciencia respecto a que esta población con AS requiere ayudas y apoyos específicos para lograr el máximo desarrollo de su potencial cognitivo o de otro tipo, y hay también un mayor conocimiento sobre los procesos de identificación (mediante la evaluación psicopedagógica) y las estrategias más 
adecuadas para dar respuesta a sus necesidades educativas. Por ello, el presente estudio centra su atención en las prácticas docentes inclusivas para atender a la población estudiantil que presenta AS y que cursa la educación secundaria en una institución educativa con modalidad de semi-internado. Este estudio cobra relevancia al exponer la autoevaluación del profesorado encargado de atender a esta población, porque podría reflejar respuestas educativas inclusivas orientadas al pleno desarrollo, aprendizaje y participación de esta población escolar.

En consonancia con lo anterior, el objetivo general de la presente investigación es analizar la autoevaluación de las prácticas inclusivas del profesorado que atiende a estudiantes con AS. Los objetivos específicos son: a) Describir la autoevaluación de las prácticas inclusivas que fortalecen el aprendizaje y la satisfacción de las necesidades educativas de estudiantes que presentan AS. b) Analizar la autoevaluación de las prácticas inclusivas que lleva a cabo el profesorado que favorece el trabajo colaborativo con la comunidad escolar. c) Comparar las diferencias de la autoevaluación de las prácticas inclusivas por sexo, nivel de estudios y de entrenamiento del profesorado que atiende al alumnado con AS.

\section{Fundamentación teórica}

\subsection{Terminología}

A lo largo de la historia, dentro del mundo de las altas capacidades, la población estudiantil ha sido comprendida de diferentes formas, derivada de las imprecisiones para unificar un concepto tan complejo, lo cual también repercute en la dificultad para identificarla y para diseñar una intervención educativa apropiada. Para efectos de esta investigación, se considera a esta población como estudiantes con AS, porque es un término conocido por el profesorado y porque se ha oficializado en México con la Propuesta de intervención educativa: Atención educativa a alumnos y alumnas con aptitudes sobresalientes (SEP, 2006), la cual los identifica como aquellas personas

[...] capaces de destacar significativamente del grupo social y educativo al que pertenecen en uno o más de los siguientes campos del quehacer humano: científicotecnológico, humanístico social, artístico o acción motriz. Estos alumnos, por presentar necesidades específicas, requieren de un contexto facilitador que les permita desarrollar sus capacidades personales y satisfacer sus necesidades e intereses para su propio beneficio y el de la sociedad. (SEP, 2006, p. 59) 
En el campo de las AS existe la creencia de que esta población sobrevive y triunfa automáticamente, que sus capacidades y habilidades se manifiestan de manera espontánea y que no requieren de apoyos específicos para superar alguna dificultad o carencia. Sin embargo, sí requieren de un acompañamiento y asesoría sistemática para satisfacer sus necesidades educativas específicas (Márquez, 2019). Para ello, es necesario que el profesorado conozca quiénes son, cómo aprende esta población, y qué posibilidades y límites ofrece la normativa en la que se sustenta la práctica educativa, basada además en los principios de atención a la diversidad.

En nuestra experiencia, cuando se aborda el tema de la educación inclusiva con el profesorado, este generalmente centra su atención en aquellos estudiantes que presentan una discapacidad, trastorno o bien otra condición que compromete sus aprendizajes. Son pocos los que consideran que la población que presenta AS enfrenta barreras para el aprendizaje y la participación en el contexto escolar y áulico. Sin embargo, el estudiantado sobresaliente no es capaz de alcanzar un desarrollo completamente autónomo por el solo hecho de serlo; por el contrario, requiere de recursos educativos, ajustes razonables y estrategias diversificadas para potenciar sus aptitudes y talentos específicos (Pérez Diez y Jiménez Fernández, 2018). De hecho, si estos estudiantes no reciben de forma oportuna una intervención educativa acorde a sus necesidades, capacidades y habilidades pueden manifestar bajo rendimiento académico o bien, una baja autoestima que repercute en su personalidad y comportamiento (Tijada, 2016). Así, la población sobresaliente no puede ser ignorada por el supuesto de que no requiere apoyos u orientaciones específicas, porque demandan entornos de aprendizaje enriquecidos, organizados por docentes que conozcan sus características, habilidades, potencialidades y necesidades educativas.

Como otros sectores de la población, el alumnado sobresaliente no es un grupo homogéneo, sino que, por el contrario, sus integrantes son tan diferentes entre sí como el resto de sus compañeros y compañeras. Sus necesidades educativas son distintas en el sentido de que pueden variar en función de factores internos, así como de los catalizadores y contextos en los que se desarrollan y aprenden. Lo cierto es que hay dos características que comparten y que les diferencian del resto de la población estudiantil: pueden aprenden más rápidamente y también pueden alcanzar mayor profundidad y extensión en el aprendizaje (Freeman, 2015). Lo anterior implica que la comunidad educativa debe tener presente la diversidad escolar y valorar esas diferencias como una oportunidad para optimizar el desarrollo no solo de los niños, niñas y adolescentes, sino de la sociedad en general. 


\subsection{Prácticas inclusivas en el aula}

Existe una variedad de estudios relacionados con las prácticas docentes inclusivas en el aula regular o en las escuelas donde se interviene a la población que presenta discapacidad o algún trastorno (de lenguaje, de aprendizaje o de conducta); sin embargo, se identifican pocos estudios que reflejen prácticas inclusivas en la atención a la población sobresaliente. Un estudio realizado por Tijada (2016) con población sobresaliente refiere la importancia de considerar metodologías centradas en el aprendizaje cooperativo, el trabajo por proyectos y programas extraescolares que permitan potenciar competencias específicas. Dichas metodologías favorecen la inclusión. Por su parte, los hallazgos de García, Belesaca y Jara (2018) refieren la importancia de actividades complementarias y extraescolares que reflejen buenas prácticas inclusivas.

Algunos estudios relacionados con prácticas inclusivas que atienden la diversidad (Darretxe, Golkietxea y Fernández, 2013; Leal y Urbina, 2014; López Melero, 2012; Rappoport y Echeita, 2018) plantean la importancia de hacer transformaciones entre la cultura institucional y el sistema de creencias del personal del colectivo docente para atender la diversidad escolar. Lo anterior también lo refiere López (2012), al puntualizar que la funcionalidad de las escuelas inclusivas está determinada por docentes que se apropian de un saber inclusivo, saber hacer inclusivo y un querer hacer inclusivo. Otros autores (Flores, Méndez y Vega, 2017; Lago, Pujolás y Naranjo, 2011; Leal y Urbina, 2014; Serrato y García, 2014) enfatizan la importancia de la formación continua, el trabajo colaborativo al interior y exterior del plantel, la vinculación con los padres de familia y, además, la preocupación del colectivo docente por apoyar los aprendizajes y la participación de todos sus estudiantes. Autores como Éste (2006) y Martínez (2002) plantean la importancia de los colectivos educativos como generadores de estrategias y prácticas pedagógicas que favorezcan la inclusión y la atención a la diversidad. Por su parte, Covarrubias y Marín (2015), García, Belesaca y Jara (2018) y González (2011) centran su interés en la formación del profesorado al puntualizar que docentes informados y capacitados pueden ayudar o contribuir de manera efectiva en los procesos de inclusión mediante una planificación adecuada, centrada en las necesidades educativas, implementando ajustes razonables, así como identificando las características individuales y el contexto de la población atendida.

Los resultados de las investigaciones revisadas reflejan que la escuela inclusiva facilita una educación pertinente, comprometida y centrada en las diferencias de la población estudiantil, justo lo que demanda la población con AS. Por ello, el profesorado requiere 
apropiarse -desde su formación inicial o como docente en ejercicio- de estrategias didácticas que apunten al desarrollo pleno del estudiantado $y$, principalmente, responder a las necesidades educativas de cada uno para garantizar un ambiente educativo inclusivo, evitando de esta manera la exclusión o la discriminación.

\section{Metodología}

\subsection{Enfoque}

Se trata de una investigación cuantitativa, transversal, con alcance descriptivo y con evaluaciones de un solo grupo (Hernández, Fernández y Baptista, 2014).

\subsection{Unidad de análisis}

Se trabajó con 34 docentes (18 hombres y 16 mujeres) que intervienen con estudiantes AS de educación secundaria en el ciclo escolar 2019-2020. Estos participantes se seleccionaron de manera no probabilística, por oportunidad.

Se gestionó ante la Secretaría de Educación del estado de Colima el permiso para acceder a las personas participantes de este estudio, quienes trabajaban en una escuela secundaria con modalidad de semi-internado, institución única en México con esta particularidad, que interviene curricularmente mediante el plan de estudio de educación secundaria vigente y de forma extracurricular oferta actividades deportivas, artísticas y culturales. Una vez obtenido el permiso correspondiente, se solicitó la participación informada y voluntaria del profesorado, aceptando el 100\%. A los participantes se les explicó con detalle el objetivo y las fases de la investigación. Después, se procedió a la firma del formato de consentimiento y, posteriormente, a la aplicación de los instrumentos a cargo de los responsables de la investigación en el marco de dos Consejos Técnicos Escolares (CTE). Durante la aplicación se aclararon dudas e inquietudes para el cumplimiento de los instrumentos, este tuvo una duración aproximada de 35 a 45 minutos en cada una de las sesiones de CTE.

\subsection{Técnicas de recolección}

Para la recolección de la información se aplicaron tres instrumentos:

a. Guía de Evaluación de Prácticas Inclusivas en el Aula Regular -GEPIA- Formato de Autorreporte (García, Romero y Escalante, 2011; García, Romero, Escalante y Flores, 2018). Esta escala evalúa aspectos considerados como fundamentales en relación con 
las prácticas inclusivas. Está conformada por 60 reactivos organizados por las siguientes subescalas: Condiciones físicas del aula, que valora los recursos áulicos con que cuentan los docentes y estudiantes y su accesibilidad; Uso del tiempo, evalúa el aprovechamiento del tiempo para actividades de aprendizaje; Metodología, explora la forma en que el profesorado toma en cuenta las características de todos sus estudiantes para organizar las actividades de aprendizaje; Evaluación, identifica si el docente realiza evaluaciones formativas, tomando en cuenta las características del alumnado; Relación docente-alumnado, evalúa la promoción del trabajo colaborativo y la gestión de un clima de aula en el que prevalece el respeto entre estudiantes y profesorado; Reflexión y sensibilización, valora las actitudes docentes hacia estudiantes; Formación docente, identifica la actualización del profesorado y sus necesidades de capacitación; Práctica del personal de apoyo y docentes, valora la cooperación entre el personal de apoyo y profesorado; y Práctica del personal de ER y familia, valora la colaboración que se establece entre docentes y familias. En los dos reactivos finales, se pide al profesorado que identifique cinco posibilidades de mejora para hacer una escuela más inclusiva y cinco temáticas que precisan para su capacitación. La GEPIA fue desarrollada para la valoración del profesorado mexicano, cuenta con valores apropiados de confiabilidad ( $\alpha=.847$ para la escala total) y validez aparente y de contenido (García et al., 2018).

b. Escala de Autoeficacia para Implementar Prácticas Inclusivas -TEIIP- (Sharma, Loreman y Forlin, 2012). Evalúa la percepción de autoeficacia del colectivo docente para implementar prácticas inclusivas en sus aulas. Está formada por 18 reactivos en escala Likert organizados en tres subescalas, los cuales, en su aplicación a docentes mexicanos, alcanzan altos niveles de confiabilidad (Alfa de Cronbach): Subescala de autoeficacia para proporcionar instrucciones inclusivas $(\alpha=.865)$, Subescala de autoeficacia para trabajar con los padres de familia y otros profesionales $(\alpha=.845)$ y la Subescala de autoeficacia para manejar conductas disruptivas $(\alpha=.855)$. La escala total tiene una confiabilidad de $\alpha=.941$ (Romero-Contreras, García-Cedillo, Forlin y LomelíHernández, 2013).

c. Estrategias para Fortalecer el Aprendizaje: Cuestionario para el profesor (2015), traducido y adaptado por Fletcher, García y Romero, basado en What really works in special and inclusive education: Using evidence-based teaching strategies (Mitchell, 2014). Este instrumento está formado por 34 reactivos en escala tipo Likert. En su aplicación a docentes mexicanos, su estructura factorial agrupó los reactivos en cinco 
subescalas: Subescala de contexto de aprendizaje, que valora el clima de aula, el aprendizaje cooperativo y la aceptación de la inclusión; Subescala de estrategias de enseñanza, que evalúa la enseñanza de estrategias cognitivas y de autorregulación; Subescala de estrategias de comportamiento, que identifica la manera en que el personal docente o la escuela manejan las conductas disruptivas de la población estudiantil; la Subescala de estrategias de recursos para el aprendizaje, que valora los recursos con que cuenta la escuela y el profesorado para su trabajo y; por último, la Subescala de cooperación interinstitucional, la cual identifica los apoyos que se proporcionan al estudiantado para potenciar sus aprendizajes. La confiabilidad de la escala total fue alta ( $\alpha=.963$ ), lo mismo que la de las subescalas (el alfa de Cronbach osciló entre $\alpha=.948$ y $\alpha=.957)$. Se hizo un análisis factorial exploratorio que validó en lo general la estructura del instrumento (Ramos-Estrada, García-Cedillo, Sotelo-Castillo, López-Valenzuela y Murillo-Parra, 2020).

\subsection{Procesamiento de análisis}

Para el análisis paramétrico apropiado y preciso utilizando el coeficiente Alpha de Cronbach que permite identificar la fiabilidad de los instrumentos, se requieren tamaños muestrales de al menos 250 individuos (Bonett, 2002), por ello se realizó dicho cálculo a nivel exploratorio y descriptivo con las respuestas de los 34 docentes involucrados. Después, se procedió al análisis de los resultados descriptivos generales con tablas de frecuencias, medias y desviaciones estándar. Para el análisis de las relaciones entre las subescalas y comparar con algunas variables sociodemográficas se utilizó el estadístico $U$ de Mann-Whitney. Todo esto usando el Software Statistical Package for the Social Sciences (SPSS v.25).

\section{Resultados}

Para obtener una perspectiva general de los resultados de los 34 docentes participantes en la investigación, debe señalarse que la media de edad de los hombres es 45.9 años ( \pm 13.7$)$, y la de las mujeres 40 años $( \pm 9.1)$. Del total de participantes, uno cursó el bachillerato técnico en artes, 22 tienen Licenciatura relacionada con la docencia y 11 cursaron Maestría. En relación con su actualización académica, 23 participantes carecen de entrenamiento para enseñar a la diversidad escolar y 11 han recibido una actualización muy escasa (menor a las 40 horas de duración). En relación con la legislación en torno a la atención a la diversidad, 23 docentes tienen un conocimiento nulo o pobre y solamente 2 tienen un conocimiento 
considerado como bueno (ninguno muy bueno). En la Tabla 1 se identifica un total de 16 participantes que mencionan tener un nivel de confianza bajo o muy bajo para enseñar a la población diversa, 6 bueno y solamente uno muy bueno (el resto un nivel promedio). Finalmente, 24 personas participantes tienen un nivel de experiencia muy bajo o bajo en la atención a la diversidad y dos participantes tienen un nivel bueno o muy bueno.

Tabla 1

Frecuencia y porcentaje del profesorado en la atención de la diversidad por dimensión según nivel, Colima-México, 2019-2020

\begin{tabular}{lllllllllll}
\hline & \multicolumn{3}{c}{ Muy bajo } & \multicolumn{2}{c}{ Bajo } & \multicolumn{4}{c}{ Promedio } & \multicolumn{3}{c}{ Bueno } & \multicolumn{2}{c}{$\begin{array}{c}\text { Muy } \\
\text { bueno }\end{array}$} \\
& $\mathrm{n}$ & $(\%)$ & $\mathrm{n}$ & $(\%)$ & $\mathrm{n}$ & $(\%)$ & $\mathrm{n}$ & $(\%)$ & $\mathrm{N}$ & $(\%)$ \\
\hline Confianza & 7 & $(21)$ & 9 & $(26)$ & 11 & $(32)$ & 6 & $(18)$ & 1 & $(3)$ \\
Experiencia & 12 & $(35)$ & 12 & $(35)$ & 8 & $(24)$ & 1 & $(3)$ & 1 & $(3)$ \\
\hline
\end{tabular}

Fuente: Elaboración propia, 2020

En relación con la Guía para Evaluar las Prácticas Inclusivas en el Aula -GEPIAFormato Autorreporte, en la Tabla 2 se observa que los puntajes de todas las subescalas oscilan entre "Casi siempre" y "Siempre" en relación con implementar las prácticas inclusivas en sus aulas (mediana total de 3.48, el rango posible va de 1 a 4). El puntaje más alto (y con menor dispersión) lo obtienen en la subescala de Evaluación (Mdn=3.83), Uso del tiempo (Mdn=3.67) y Metodología (Mdn=3.61), seguida de las subescalas de Reflexión y sensibilización (mediana de 3.60) y Relación docente-estudiantes y Planeamiento (ambas con 3.50). Las subescalas con el menor puntaje son Práctica del personal de apoyo y Formación docente (ambas con Mdn=3.25).

Tabla 2

Medianas, medias y desviaciones estándar del profesorado, Colima-México, 2019-2020

\begin{tabular}{lccc}
\hline \multirow{2}{*}{ Subescalas GEPIA y Escala Total } & \multicolumn{3}{c}{ Docentes (n= 34) } \\
\cline { 2 - 4 } & Mediana & Media & Desviación estándar \\
\hline Condiciones físicas del aula & 3.40 & 3.47 & 0.43 \\
Planeamiento & 3.50 & 3.56 & 0.36 \\
Uso del tiempo & 3.67 & 3.56 & 0.42 \\
Metodología & 3.61 & 3.57 & 0.37 \\
Evaluación & 3.83 & 3.64 & 0.19 \\
Relación docente-estudiantes & 3.50 & 3.45 & 0.39 \\
Práctica del personal de apoyo & 3.33 & 3.20 & 0.68 \\
Reflexión y sensibilización & 3.60 & 3.65 & 0.25 \\
Formación docente & 3.25 & 3.30 & 0.47 \\
Práctica del personal de apoyo y docentes & 3.25 & 3.19 & 0.53 \\
Práctica del personal de ER y familias & 3.33 & 3.17 & 0.86 \\
Escala total & 3.48 & 3.46 & 0.61 \\
\hline
\end{tabular}

Fuente: Elaboración propia a partir de los resultados de análisis, 2020 
De las subescalas con puntaje alto, la de Evaluación implica la realización de evaluaciones continuas y formativas, usar la evaluación para motivar a estudiantes y tomar en cuenta sus aprendizajes previos. Asimismo, con puntajes altos se identifican las subescalas Uso del tiempo y Planeamiento, estando muy relacionadas, puesto que la primera valora si el profesorado optimiza el tiempo disponible en el aula para motivar al alumnado y para realizar actividades académicas; por su parte, la segunda valora el planeamiento de las actividades de enseñanza considerando los aprendizajes a lograr, la evaluación y los ritmos y estilos de aprendizaje de la población estudiantil. Reflexión y sensibilización se relaciona con evitar las prácticas discriminatorias en el grupo, y tomar en cuenta a la población estudiantil al momento de realizar las actividades, además de que los profesores reflexionen sobre la eficacia de sus prácticas. Igualmente, con puntajes altos, aparece la subescala de Metodología que, teóricamente, es de las más importantes e indica si el colectivo docente conoce a todos sus estudiantes y ajusta su metodología de enseñanza para lograr que todos aprendan (incluye la realización de adecuaciones curriculares para educandos con NEE). Por otra parte, el profesorado puede mejorar en lograr una colaboración más efectiva del personal de apoyo (subescala Personal de apoyo) buscando realizar trabajo colaborativo con este (subescala Práctica del personal de apoyo y docentes) y en el conocimiento y colaboración con las familias de todos sus estudiantes (Práctica del personal de ER y familias). Las subescalas mencionadas son las que obtuvieron los puntajes más bajos y la mayor dispersión.

En los resultados de las preguntas abiertas de la GEPIA, centradas en los aspectos que deben ser mejorados por parte de la escuela y del profesorado para ofrecer una educación inclusiva, el $80 \%$ de las personas participantes en el estudio señala la necesidad de capacitación, 65\% habla de la insuficiencia de materiales y equipo, $62 \%$ indica que es necesario la mejora de infraestructura, 50\% menciona la necesidad de apoyo de especialistas para atender las aptitudes sobresalientes, 35\% del trabajo colaborativo y 14\% la inclusión y coordinación de toda la comunidad escolar.

Por otra parte, todo el cuerpo docente refiere que las temáticas para su formación, capacitación y/o actualización deben ser variadas y acordes a sus necesidades pedagógicas. Las más señaladas fueron el tema de inclusión educativa (estrategias para diversificar la enseñanza e identificación y atención a las AS) con 38\% y la necesidad de enfocarse en contenidos relacionados con las aptitudes sobresalientes y necesidades educativas especiales (NEE) con un $29 \%$. Un $24 \%$ en la implementación de la tecnología que permita el acercamiento o la mejora de la práctica pedagógica, 18\% considera importantes los temas sobre liderazgo, 
$12 \%$ indica la necesidad de cursos sobre convivencia, motivación y valores, y el $9 \%$ señala la necesidad de analizar temas relacionados con los derechos humanos y la ética en la docencia. Este hallazgo de formación, capacitación y actualización coincide con estudios realizados por Covarrubias y Marín (2015), García, Belesaca y Jara (2018) y González (2011) al puntualizar que el profesorado informado y capacitado contribuye de manera efectiva en los procesos de inclusión por planificaciones centradas en ajustes razonables y metodologías diversas que responden a las necesidades educativas del alumnado.

En la Tabla 3 se presentan los puntajes de la Escala de Autoeficacia para Implementar Prácticas Inclusivas -TEIIP-, donde se aprecia que el colectivo docente obtiene puntajes promedio altos (el rango va de 1 a 6 ). Con respecto a la subescala de Eficacia en el manejo de la conducta, el profesorado se auto percibe capaz de comunicar sus expectativas en cuanto a las conductas de sus estudiantes, de prevenir y controlar las conductas disruptivas 0 agresivas, y de hacer que acaten las reglas. Con respecto a la subescala de Eficacia en la colaboración, el profesorado se valora capaz de hacer que las familias se sientan cómodas al apoyar y trabajar colaborativamente, de trabajar colaborativamente con otros profesionales y de informar apropiadamente sobre leyes y normas relacionadas con la educación inclusiva. Finalmente, de acuerdo con la subescala de Eficacia para la enseñanza inclusiva, se evidencia que el profesorado se percibe relativamente capaz de comprender lo que sus educandos están aprendiendo, de retarlos académicamente, de promover el trabajo colaborativo, usar distintas estrategias de evaluación y de buscar opciones para explicar mejor los contenidos.

Tabla 3

Medianas, medias y desviaciones estándar del profesorado, Colima-México, 2019-2020

\begin{tabular}{lccc}
\hline Escalas y subescalas & Mediana & Media & $\begin{array}{c}\text { Desviación } \\
\text { estándar }\end{array}$ \\
\hline $\begin{array}{l}\text { Escala de Autoeficacia para Implementar Prácticas } \\
\text { Inclusivas -TElIP- }\end{array}$ & 4.81 & 4.70 & 1.12 \\
\hline Subescala de eficacia para la enseñanza inclusiva & 4.83 & 4.09 & 0.96 \\
Subescala de eficacia en el manejo de la conducta & 5.00 & 4.70 & 1.13 \\
Subescala de eficacia en la colaboración & 4.83 & 4.63 & 1.12
\end{tabular}

Fuente: Elaboración propia a partir de los resultados de análisis, 2020

A la luz de los resultados de la Escala de Autoeficacia para Implementar Prácticas Inclusivas -TEIIP- el profesorado obtiene puntajes medianos altos al promover acciones de colaboración con toda la comunidad escolar. Estos hallazgos coinciden con lo que especifican (Flores, Méndez y Vega, 2017; Lago, Pujolás y Naranjo, 2011; Leal y Urbina, 2014; Serrato y 
García, 2014) al enfatizar la importancia del trabajo colaborativo al interior y exterior del plantel, así como la vinculación con los padres y las madres de familia.

A continuación, se presentan, en la Tabla 4, los puntajes correspondientes al Cuestionario de Estrategias para Fortalecer el Aprendizaje. La escala total muestra a docentes que se evalúan de una manera moderada en las estrategias que implementan en el aula (el rango de puntajes va de 1 a 4). En la subescala Contexto de aprendizaje, el profesorado obtiene el puntaje más alto, lo que implica que con frecuencia organizan a sus estudiantes en equipos o triadas para fomentar el trabajo cooperativo, buscan la cooperación con las familias y otros profesionales, cuentan con un entorno apropiado generando buen clima de aula. Asimismo, tienen un compromiso con la educación inclusiva y adaptan sus evaluaciones para hacerlas apropiadas a todos sus educandos. Sus Estrategias del comportamiento, también son relativamente altas, lo que refleja una enseñanza centrada en el desarrollo de habilidades sociales y afectivas por la implementación de actividades extracurriculares para prevenir y reducir conductas indeseadas de sus estudiantes. Por su parte, las subescalas de Estrategias de cooperación interinstitucional y Estrategias de enseñanza son moderadas, la primera indica que el profesorado hace un esfuerzo por ajustar sus prácticas para satisfacer las necesidades académicas y afectivas de todos sus estudiantes mediante el diseño universal de aprendizaje, adecuaciones curriculares y un seguimiento cercano; mientras la segunda implica que el colectivo docente procura apoyar a sus estudiantes mediante repasos, estrategias cognitivas, a mejorar su comprensión lectora y a que definan sus metas. Finalmente, el colectivo docente obtiene un puntaje bajo en la subescala Estrategias de recursos para el aprendizaje, que implica que no cuentan con el equipo apropiado ni con facilidades de acceso para atender a estudiantes con discapacidad y que tampoco cuentan con suficientes apoyos de personal especializado.

Tabla 4

Medianas, medias y desviaciones estándar del profesorado, Colima-México, 2019-2020

\begin{tabular}{lcccc}
\hline \multicolumn{2}{l}{ Escalas y subescalas } & Mediana & Media & $\begin{array}{c}\text { Desviación } \\
\text { estándar }\end{array}$ \\
\hline $\begin{array}{l}\text { Cuestionario Estrategias para fortalecer } \\
\text { aprendizaje }\end{array}$ & el & $\mathbf{3 . 1 0}$ & $\mathbf{3 . 1 2}$ & $\mathbf{0 . 8 0}$ \\
\hline $\begin{array}{l}\text { Contexto de aprendizaje } \\
\text { Estrategias de enseñanza }\end{array}$ & 3.35 & 3.27 & 0.84 \\
Estrategias del comportamiento & 3.30 & 3.19 & 0.71 \\
Estrategias de cooperación interinstitucional & 3.30 & 3.26 & 0.70 \\
Estrategias de recursos para el aprendizaje & 3.10 & 3.21 & 0.76 \\
\hline
\end{tabular}

Fuente: Elaboración propia con información de las personas participantes, 2020 
El resultado de la escala total del Cuestionario de Estrategias para Fortalecer el Aprendizaje muestra que el profesorado implementa una moderada aplicación de estrategias para promover los saberes del alumnado sobresaliente. Sin embargo, aun cuando se autoevalúan de esta forma, se identifican prácticas inclusivas que coadyuvan a potencializar habilidades cognitivas, afectivas y de colaboración con la población AS. Este hallazgo tiene estrecha relación con lo que puntualizan Pérez y Jiménez (2018) al señalar que el alumnado sobresaliente demanda de estrategias diversificadas y recursos educativos para el logro de un aprendizaje autónomo y significativo.

De acuerdo con Bonett (2002) si el tamaño de muestra es demasiado pequeño el cálculo del Alpha de Cronbach carecerá de potencia y el intervalo de confianza será demasiado alto. Bonett y Wright (2014) señala que si se estima el Alfa de Cronbach para una muestra muy pequeña el resultado puede parecer impresionante, pero si se analiza el intervalo de confianza este podría indicar una confiabilidad muy baja. Es por esto que, con la finalidad de describir de manera exploratoria la confiabilidad de las escalas y las subescalas de los instrumentos aplicados, se realizó un análisis mediante el Alpha de Cronbach, sin embargo, cabe aclarar que la muestra participante en el estudio solo fue de 34 individuos por lo que, dado lo señalado anteriormente; los resultados que se presentan a continuación deben tomarse con precaución. El puntaje total de la GEPIA ( $\alpha=0.914)$ indica una confiabilidad excelente, para las subescalas de Práctica del personal de educación regular y familias $(\alpha=0.847)$, Metodología $(\alpha=0.837)$, Relación docente-estudiante $(\alpha=0.816)$ y Evaluación $(\alpha=0.809)$ señalan una confiabilidad buena; Práctica del personal de apoyo ( $\alpha=0.777)$, Planeamiento $(\alpha=0.776)$, Condiciones físicas del aula $(\alpha=0.725)$, Práctica del personal de apoyo y docentes de grupo $(\alpha=0.657)$ y, Formación docente ( $\alpha=0.553$ ) tiene una confiabilidad menor pero, de acuerdo con Nunnally (1978), aún aceptable. Sin embargo, los resultados de la subescala de Reflexión y sensibilización $(\alpha=0.272)$ deben tomarse con reservas dado que tienen valores pobres de confiabilidad.

Si se tratara de una muestra adecuada, de acuerdo con Bonett (2002) conformada por al menos 250 individuos, los resultados de la TEIIP se encuentran buenos valores de confiabilidad en las Subescala de Eficacia para la Enseñanza Inclusiva ( $\alpha=0.890)$, Subescala de Eficacia en el Manejo de Conducta $(\alpha=0.873)$ y Subescala de Eficacia en la Colaboración $(\alpha=0.886)$ y resultados excelentes en el Alpha de Cronbach de la escala total $(\alpha=0.952)$. En lo que refiere al Cuestionario de Estrategias para fortalecer el aprendizaje, se encontró una confiabilidad excelente en general $(\alpha=0.923)$ y buena en las subescalas de Contexto de aprendizaje $(\alpha=0.808)$, Estrategias de enseñanza $(\alpha=0.804)$, Estrategias de recursos para el 
aprendizaje $(\alpha=0.746)$ y aunque menores, pero aun aceptables, en las subescalas Estrategias de cooperación interinstitucional $(\alpha=0.660)$ y Estrategias del comportamiento $(\alpha=0.587)$.

En la búsqueda del efecto de algunas variables independientes como el sexo, nivel de estudios y nivel de entrenamiento en las percepciones del profesorado sobre la inclusión, dado el tamaño de la muestra, se aplicó el estadístico $U$ de Mann-Whitney en cada una de las escalas, con sus respectivas subescalas y se aceptó el 95\% de confianza. A continuación, se presentan solo los datos donde se encontraron diferencias estadísticamente significativas.

En lo que refiere a la GEPIA se encontraron diferencias estadísticamente significativas entre hombres y mujeres $(\mathrm{U}(32)=80.5, \mathrm{p}=0.018)$ en la subescala Evaluación, donde los hombres respondieron implementar con mayor frecuencia prácticas inclusivas en las aulas $(\mathrm{Mdn}=4.0)$ en comparación con las mujeres ( $\mathrm{Mdn}=3.5)$. También se encontraron diferencias estadísticamente significativas entre los niveles de entrenamiento $(U(32)=63.5, p=0.018)$, en la subescala Práctica del personal de apoyo y docentes de grupo donde el profesorado con nulo entrenamiento señala implementar con mayor frecuencia prácticas inclusivas en el aula $(\mathrm{Mdn}=3.25)$ en comparación con docentes con un entrenamiento escaso ( $\mathrm{Mdn}=3.00)$. Finalmente, se encontraron diferencias estadísticas altamente significativas en la subescala Condiciones físicas del aula $(\mathrm{U}(32)=44, \mathrm{p}=0.002)$ donde el profesorado con nulo entrenamiento otorga respuestas mayores en la escala $(\mathrm{Mdn}=3.6)$ en comparación con aquellos que tenían un entrenamiento escaso ( $\mathrm{Mdn}=3.2)$.

Los resultados otorgados a la TEIIP no presentan diferencias estadísticamente significativas entre sexo, nivel de estudios o nivel de entrenamiento. En cambio, en el Cuestionario Estrategias para Fortalecer el Aprendizaje, específicamente en la subescala de las Estrategias de cooperación interinstitucional, se encontraron diferencias estadísticamente significativas $(\mathrm{U}(32)=85.5, \mathrm{p}=0.042)$ entre ambos sexos, ya que los hombres otorgaron en promedio puntajes superiores ( $M d n=3.4)$ a los de las mujeres ( $M d n=3.0)$, lo que significa que los docentes consideran que se esfuerzan más por atender las necesidades educativas específicas de sus estudiantes mediante la adaptación del currículo, diseño universal del aprendizaje y seguimiento cercano. Además, se presentan diferencias significativas $(\mathrm{U}(32)=68, \mathrm{p}=0.008)$ en la subescala de Estrategias de recursos para el aprendizaje, donde los puntajes de los hombres $(\mathrm{Mdn}=2.8)$ son mayores a los de las mujeres $(\mathrm{Mdn}=2.0)$. Es decir, son las mujeres quienes consideran mayoritariamente que no cuentan con el equipo apropiado ni con facilidades de acceso para atender a los educandos, ni con suficientes apoyos de personal especializado. 
Considerando los resultados globales de la presente investigación, se identifica una diversidad de buenas prácticas inclusivas del colectivo docente que labora en la Institución, donde intervienen con estudiantes de educación secundaria que presentan aptitudes sobresalientes. La autoevaluación de prácticas inclusivas que realiza el profesorado muestra una actitud positiva hacia los principios de la educación inclusiva, lo que se traduce en la evitación de prácticas discriminatorias en el grupo, ya que toma en cuenta a todo el estudiantado al momento de realizar las actividades y evaluar el proceso formativo ajustando su metodología de enseñanza para lograr que todos aprendan.

Estos resultados están en la misma línea de los encontrados en Tijada (2016) con población sobresaliente, al referir metodologías que permiten potenciar competencias específicas y favorecer la inclusión. Además, debe tomarse en cuenta que la planta docente fue seleccionada para trabajar en esta institución por haberse destacado de manera significativa en su práctica docente en otras instituciones.

Las derivaciones de los estudios realizados por Flores, Méndez y Vega (2017), Lago, Pujolás y Naranjo (2011), Leal y Urbina (2014) y Serrato y García (2014) destacan la importancia del trabajo colaborativo al interior y exterior del plantel y la vinculación con padres de familia. Asimismo, ponderan alto el trabajo en equipo con sus educandos a través de proyectos o actividades extracurriculares. Los hallazgos de la Escala de Autoeficacia para Implementar Prácticas Inclusivas -TEIIP- tienen resultados similares a los de las investigaciones antes citadas, al identificar que el profesorado se distingue por emprender acciones para lograr que las familias se sientan cómodas y en confianza para emprender tareas y/o gestiones en conjunto con ellos.

De igual manera sucede con el equipo de profesionales que labora en la Institución, porque implica estar coordinados para responder no solo a las necesidades educativas, sino también a las sociales y a las relacionadas con la salud e integridad del estudiantado, esto debido a la modalidad de semi-internado que opera la secundaria. Por otra parte, se evidencia que el profesorado planifica y lleva a cabo actividades académicas centradas en retos cognitivos, organiza el trabajo en equipos para fomentar el trabajo colaborativo promoviendo un entorno inclusivo. Otro rasgo importante identificado, es que el colectivo docente procura apoyar a sus estudiantes a mejorar su comprensión lectora y definir sus metas mediante repasos y estrategias cognitivas.

No cabe duda que la pieza fundamental del engranaje de buenas prácticas inclusivas es el profesorado. El colectivo docente puede ser un facilitador o representar una barrera para el 
aprendizaje y la participación de sus estudiantes. Los participantes de la presente investigación mostraron prácticas educativas inclusivas que promueven el trabajo en equipo con la comunidad escolar, especialmente la participación plena y efectiva de los padres de familia y el alumnado.

Un rasgo relevante en los hallazgos del Cuestionario de Estrategias para Fortalecer el Aprendizaje es que, en comparación con otras estrategias para lograr la inclusión, al profesorado le implica un mayor esfuerzo atender las necesidades académicas del alumnado. Esta situación es posible que se relacione con la falta de trayectos de capacitación y/o actualización con temas relacionados con la educación inclusiva y las aptitudes sobresalientes, reflejando un área de oportunidad importante de atender. Como menciona González (2011) al referir que docentes bien capacitados pueden ayudar o contribuir de manera efectiva en los procesos de inclusión educativa al tener elementos teóricos metodológicos para atender a la diversidad escolar.

Con referencia a lo anterior, la falta de capacitación (que también se identifica en las respuestas abiertas de la GEPIA) en temas relacionados con la educación inclusiva, las aptitudes sobresalientes y la atención a la diversidad, se trata de contenidos poco abordados al interior de la escuela y cobran relevancia para implementar prácticas inclusivas y adquirir competencias que mejoren el desempeño de la planta docente en su trabajo al interior de las aulas, especialmente con la población AS. La comunidad escolar debe estar suficientemente preparada para atender la diversidad escolar, especialmente en el diseño y aplicación de estrategias innovadoras en beneficio de ambientes educativos inclusivos.

Debe dimensionarse la cuestión relacionada con la falta de capacitación. De acuerdo con la encuesta de Talis, los docentes mexicanos demandan mayor capacitación en temas relacionados con el manejo de las Tecnologías de la Información y la Comunicación, la enseñanza en entornos multiculturales/multilingües y una necesidad todavía mayor en la enseñanza de alumnos con necesidades educativas especiales (Organización para la Cooperación y el Desarrollo Económico [OCDE], 2019).

El avance hacia culturas más inclusivas en toda institución educativa es un proceso que toma tiempo, ya que es el resultado de la transformación de sus políticas educativas y de la apropiación de prácticas educativas innovadoras e inclusivas por parte del colectivo docente. Sin embargo, un rasgo que se identifica en el Cuestionario de Estrategias para Fortalecer el Aprendizaje, como buena práctica inclusiva es la relacionada con la enseñanza de habilidades sociales, ya que el profesorado cuenta con programas para prevenir y reducir comportamientos 
indeseados de sus estudiantes (esto también se puede observar en la GEPIA), lo que favorece el clima de aula y, por tanto, la inclusión social.

Otro hallazgo relevante, aunque con puntaje bajo, es el que refiere el colectivo docente con mayor experiencia, al especificar que no cuenta con el equipo apropiado ni con las suficientes facilidades de acceso para atender a educandos con aptitud sobresaliente, además de que no cuentan con suficientes apoyos de personal especializado. Esto se ve reflejado en la Subescala de estrategias de recursos para el aprendizaje del Cuestionario de Estrategias para Fortalecer el Aprendizaje. Evidentemente, es imprescindible que el profesorado cuente con el material y las herramientas técnicas y metodológicas para atender la diversidad.

\section{Conclusiones}

En la Constitución Política de los Estados Unidos Mexicanos (Cámara de Diputados del H. Congreso de la Unión de México, 2019a), la educación es un derecho cobijado por los principios de equidad, igualdad y excelencia educativa. Bajo estos preceptos, las prácticas pedagógicas deben mejorar para eliminar o minimizar las barreras que limitan el aprendizaje y la participación del alumnado, mismas que surgen de la interacción entre estudiantes y su contexto educativo, así como de las políticas y culturas.

El presente estudio alcanzó el objetivo planteado, centrado en analizar la autoevaluación de prácticas inclusivas docentes que interviene con adolescentes que presentan aptitudes sobresalientes. Se identificó actitud positiva por parte del profesorado hacia los principios de la educación inclusiva, la implementación de estrategias diversificadas en la metodología y la evaluación, la aplicación de retos cognitivos, así como el trabajo colaborativo con la comunidad escolar y la vinculación con padres de familia. Conocer las prácticas inclusivas del colectivo docente de la escuela secundaria es interesante por las siguientes razones: porque es única en el país en la modalidad de semi-internado, atiende exclusivamente a estudiantes con aptitudes sobresalientes, tiene un costo económico alto por las actividades curriculares y extracurriculares que oferta, se interviene con grupos reducidos, cuenta con un equipo multidisciplinario y desarrolla el método por proyectos. En sí, implementa prácticas de discriminación positiva al generar estrategias educativas diversificadas para potencializar las habilidades cognitivas, académicas, sociales, artísticas y deportivas de la población que atiende.

Reflexionamos que, si las escuelas regulares y especiales, que atienden a estudiantes con discapacidad o trastorno, tuvieran los mismos recursos y el mismo tipo de profesores 
participantes en este estudio, sus resultados serían mucho mejores de los que actualmente obtienen, por lo cual serían muy atractivas para los estudiantes con y sin discapacidad. Esto es interesante porque permite analizar la polémica relacionada con las escuelas regulares y especiales con un nuevo enfoque: el relacionado con los recursos. En otras palabras, si todas las escuelas contaran con los mismos recursos con los que cuenta la Institución que participó en la presente investigación (una buena dirección, docentes muy motivados y capaces de responder a las necesidades de todos sus estudiantes, recursos económicos, posibilidades de gestión institucional, actividades extraescolares y apoyo de padres y madres de familia), la educación en el país tendría el impulso para alcanzar la calidad que toda la sociedad les demanda.

Algunas de las principales limitaciones que tiene la presente investigación se relacionan con la falta de implementación de la técnica de observación en el aula para identificar las prácticas inclusivas del profesorado; es decir, el análisis de registros de observación con el cumplimiento de los instrumentos implementados. Otra de las limitaciones es la dificultad de escuchar y dar voz al colectivo docente que interviene con la población AS en aulas regulares. Por ello, resulta necesario crear estrategias o herramientas para que, a pesar de que no se cuenta con la posibilidad de identificar a todo el profesorado en una sola Institución (están dispersos a lo largo del sistema educativo), se conozcan sus percepciones en torno a la educación inclusiva para contrastarlos con los resultados del presente estudio.

La educación inclusiva cultiva transformaciones en las escuelas y en especial, en las aulas porque induce al trabajo conjunto de toda la comunidad educativa: equipo directivo, docentes, estudiantes y familias. Por ello, valdría la pena apostar por un colectivo escolar que sea generador de una autonomía de gestión que impacte de manera positiva en las prácticas inclusivas y que repercuta principalmente en satisfacer las necesidades académicas del alumnado.

Para finalizar, especulamos hacer un alto en el camino para reflexionar sobre las prácticas inclusivas en las aulas, una reflexión que permita valorar positivamente la diversidad y que conduzca a la mejora educativa. La población estudiantil es diversa, diferente y única, lo que obliga a la movilización, a pensar y replantear las acciones para lograr un aprendizaje equitativo y de calidad, porque ante todo está el interés superior de la niñez. Sin embargo, se debe reconocer que aún falta mucho por hacer. Este, el de la implementación de la educación inclusiva, es tarea de todos y todas. 


\section{Agradecimientos}

Agradecimiento especial a la comunidad escolar de la escuela secundaria que interviene con estudiantes que presentan aptitudes sobresalientes.

\section{Referencias}

Blanco, Rosa. (2006). La equidad y la inclusión social: uno de los desafíos de la educación y la escuela hoy. Revista Electrónica Iberoamericana sobre Calidad, Eficacia y Cambio en Educación, 4(3), 1-15. Recuperado de https://www.redalyc.org/articulo.oa?id=55140302

Bonett, Douglas G. (2002). Sample size requirements for testing and estimating coefficient alpha. Journal of educational and behavioral statistics, 27(4), 335-340. Recuperado de https://bit.ly/3sHEJfR

Bonett, Douglas G., and Wright, Thomas. A. (2014). Cronbach's alpha reliability: Interval estimation, hypothesis testing, and sample size planning. Journal of Organizational Behavior, 36(1), 315. Recuperado de https://bit.ly/3ayv1Gk

Booth, Tony., y Ainscow, Mel. (2015). Guía para la Educación Inclusiva. Desarrollando el aprendizaje y la participación en los Centros Escolares. Madrid, España: FUHEM. Recuperado de https://downgalicia.org/wp-content/uploads/2018/01/Guia-para-laEducacion-Inclusiva.pdf

Cámara de Diputados del H. Congreso de la Unión de México. (2019a). Constitución política de los Estados Unidos Mexicanos. Recuperado de http://www.diputados.gob.mx/LeyesBiblio/pdf mov/Constitucion Politica.pdf

Cámara de Diputados del H. Congreso de la Unión. (2019b). Ley General de Educación. Recuperado de http://www.diputados.gob.mx/LeyesBiblio/pdf/LGE 300919.pdf

Covarrubias Pizarro, Pedro., y Marín Uribe, Rigoberto. (2015). Evaluación de la propuesta de intervención para estudiantes sobresalientes: caso Chihuahua, México. Revista Actualidades Investigativas en Educación, 15(3), 1-32. doi: http://dx.doi.org/10.15517/aie.v15i3.19457

Derretxe Urrutxi, Leire, Goikoetxea Piédrola, Javier y Fernández González, Almudena. (2013). Análisis de prácticas inclusivas y exclusoras en dos centros educativos del país Vasco. Revista electrónica Actualidades Investigativas en Educación, 13(2), 1-30.Recuperado de https://www.redalyc.org/articulo.oa?id=44727049005

Flores Jasso, Andrea., Méndez Pineda, Juana., y Vega Martínez, Blanca. (2017). Prácticas inclusivas como estrategia de cambio: construyendo una escuela para todos. Revista Educando para Educar, 18(33), 19-28. Recuperado de https://beceneslp.edu.mx/ojs2/index.php/epe/article/view/9/9

Freeman, Joan. (2015). Por qué algunos niños con altas capacidades son notablemente más exitosos en la vida que otros con iguales oportunidades y habilidad. Revista de Educación, (368), 255-278. Recuperado de https://sede.educacion.gob.es/publiventa/detalle.action?cod=20329 
García Cedillo, Ismael., Romero Contreras, Silvia., y Escalante Aguilar, Liliana. (2011). Diseño y validación de la guía de evaluación de las prácticas inclusivas en el aula (GEPIA). En $X I$ Congreso nacional de investigación educativa. Universidad Autónoma de México, México. Recuperado http://www.comie.org.mx/congreso/memoriaelectronica/v11/docs/area 14/1390.pdf

García Cedillo, Ismael., Romero Contreras, Silvia., Escalante Aguilar, Liliana., y Flores Barrera, Vasthi. (2018). Algunas propiedades psicométricas de las guías para evaluar prácticas inclusivas en el aula. Revista Española de Orientación y Psicopedagogía, 29(2), 8-28. Recuperado de http://revistas.uned.es/index.php/reop/article/view/23150/18558

García Guevara, Wilson, Belesaca, Oscar y Jara Saldaña, Gabriela. (2018). Prácticas inclusivas de los docentes. Killkana Sociales: revista de investigación científica, 2(2), 2530. doi: https://doi.org/10.26871/killkana social.v2i2.303

González Fernández, Raúl. (2011). Actitud del profesorado respecto a la integración e inclusión en el tratamiento educativo de la diversidad (Tesis de doctorado). Universidad Nacional de Educación a Distancia, España. Recuperado de https://dialnet.unirioja.es/servlet/tesis?codigo=220143

Hernández Sampieri, Roberto, Fernández Collado, Carlos y Baptista Lucio, María del Pilar. (2014). Metodología de la investigación (6ª ed). México: McGraw-Hill.

Juárez Núñez, José, Comboni Salinas, Sonia y Garnique Castro, Fely. (2010). De la educación especial a la educación inclusiva. Argumentos, 23(62), 41-83. Recuperado de http://www.scielo.org.mx/pdf/argu/v23n62/v23n62a3.pdf

Lago, José Ramón, Pujolás, Pere y Naranjo, Mila. (2011). Aprender cooperando para enseñar a cooperar: procesos de formación/asesoramiento para el desarrollo del Programa CA/AC. Aula: Revista de Pedagogía de la Universidad de Salamanca, (17),89-106. Recuperado de https://revistas.usal.es/index.php/0214-3402/article/view/8397/9107

Leal Leal, Karen y Urbina Cárdenas, Jesús. (2014). Las prácticas pedagógicas y la inclusión educativa. Revista Latinoamericana de Estudios Educativos, 10(2), 11-33. Recuperado de http://190.15.17.25/latinoamericana/downloads/Latinoamericana10(2) 2.pdf

López Melero, Miguel. (2012). La escuela inclusiva: una oportunidad para humanizarnos. Revista Interuniversitaria de Formación del Profesorado, 26(2), 131-160. Recuperado de https://www.redalyc.org/pdf/274/27426890007.pdf

Márquez Cabellos, Norma Guadalupe. (2019). Atención educativa a las aptitudes sobresalientes: propuesta didáctica a través de estudio de casos. En J. Cuevas Romo y A. Trejo Larios. Secuencias didácticas para el aprendizaje de las ciencias y las matemáticas. México: CENEJUS-UASLP.

Martínez Geijo, Pedro. (2002). Categorización de comportamientos de enseñanza desde un enfoque centrado en los estilos de aprendizaje (Tesis doctoral). Universidad Nacional de Educación a Distancia, España. Recuperado de https://dialnet.unirioja.es/servlet/tesis?codigo=38618 
Mitchell, David. (2014). Estrategias para fortalecer el aprendizaje: Cuestionario para el profesorado (2015). En: What really works in special and inclusive education: Using evidence-based teaching strategies ( $2^{\mathrm{a}}$ ed). New York, USA: Routledge. Recuperado de https://www.academia.edu/37967852/David Mitchell 2008 What really Works in Usi ng Evidence based Teaching Strategy

Nunnally, Jum. (1978). Psychometric theory. New York: McGraw-Hill.

Organización para la Cooperación y Desarrollo Económicos (OCDE). (2019). Resultados de Talis 2018. Volume I: Teachers and School Leaders as Lifelong Learners. Recuperado de https://www.oecd.org/education/talis/TALIS2018 CN MEX es.pdf

Orozco, Inmaculada y Moriña, Anabel. (2019). Prácticas docentes para una pedagogía inclusiva en educación primaria: Escuchando las voces del profesorado. Aula Abierta, 48(3), 331-338. doi: https://doi.org/10.17811/rifie.48.3.2019.331-338

Pérez Diez, Lorenzo y Jiménez Fernández, Carmen. (2018). Influencia de la organización escolar en la educación de los alumnos de altas capacidades. Enseñanza \& Teaching, 36(1), 151-178. doi: https://doi.org/10.14201/et2018361151178

Ramos-Estrada, Dora., García-Cedillo, Ismael., Sotelo-Castillo, Mirsha., López-Valenzuela, Mercedes., y Murillo-Parra, Luisa. (2020). Validación de un instrumento de estrategias para fortalecer el aprendizaje. Revista Electrónica Educare, 24(1), 1-15. doi: http://doi.org/10.15359/ree.24-1.6

Rappoport Redondo, Soledad y Echeita, Gerardo. (2018). El docente, los profesionales de apoyo y las prácticas de enseñanza: aspectos claves en la configuración de aulas inclusivas. Perspectiva educacional: Formación de profesores, 57(3), 3-27. doi: http://dx.doi.org/10.4151/07189729-Vol.57-Iss.3-Art.740

Romero-Contreras, Silvia., García-Cedillo, Ismael., Forlin, Chris., y Lomelí-Hernández, Karla (2013). Preparing teachers for inclusion in Mexico: how effective is this process? Journal of Education for Teaching: International Research and Pedagogy, 39(5). doi: https://doi.org/10.1080/02607476.2013.836340

Secretaría de Educación Pública [SEP]. (2006). Propuesta de intervención: Atención educativa a alumnos y alumnas con aptitudes sobresalientes. México: SEP. Recuperado de https://elisaee.files.wordpress.com/2013/03/propuesta-de-intevenciefbfbdn.pdf

Secretaría de Educación Pública [SEP]. (2019). Acuerdo Educativo Nacional. Estrategia Nacional de Educación Inclusiva. México: SEP

Serratos Almendárez, Lilia y García Cedillo, Ismael. (2014). Evaluación de un programa de intervención para promover prácticas docentes inclusivas. Revista Actualidades Investigativas en Educación,14(3), 1-25. Recuperado de https://www.redalyc.org/pdf/447/44732048015.pdf 
Sharma, Umesh., Loreman, Tim., y Forlin, Chris. (2012). Measuring teacher efficacy to implement inclusive practices. Journal of Research in Special Educational Needs, 12(1), 12-21. doi: https://doi.org/10.1111/1.1471-3802.2011.01200.x

Tijada Inés, Patricia. (2016). Las altas capacidades en la escuela inclusiva. Revista internacional de apoyo a la inclusión, logopedia, sociedad y multiculturalidad, 2(1), 7588. Recuperado de https://revistaselectronicas.ujaen.es/index.php/riai/article/view/4196/3421 
Revista indizada en

scip/o redalyc latindex

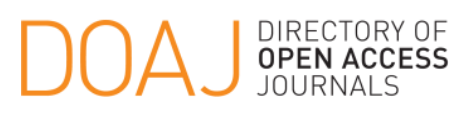

Distribuida en las bases de datos:

- Dialnet

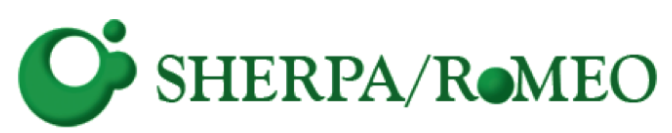

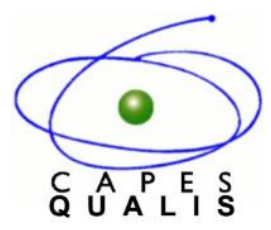

MIAR 